\title{
Hyperholomorphic functions on commutative algebras
}

\author{
ANATOLIY A. POGORUI* \\ Department of Mathematics, Zhytomyr State University, \\ Zhytomyf, 0008, Ukraine \\ Communicated by R. P. Gilbert
}

(Received 14 February 2007; in final form 4 July 2007)

\begin{abstract}
In this article, we study properties of hyperholomorphic functions on commutative finite-dimensional algebras. The Cauchy-Riemann type conditions for hyperholomorphic functions is investigated. We prove that a hyperholomorphic function on a commutative finitedimensional algebra can be expanded in a Taylor series. We also present a technique for computing zeros of polynomials in commutative algebras.
\end{abstract}

Keywords: Hyperholomorphic function; Commutative algebra; Polynomial

AMS Subject Classifications: Primary 32A10; Secondary 13M10

\section{Introduction}

The development of hyperholomorphic function analysis has renewed interest in mathematics and physics because of fruitful applications. One of the most popular hypercomplex analysis is quaternionic analysis; however, noncommutativity of quaternion algebra causes many intractable problems, for instance, the problem of expansion of a hyperholomorphic quaternionic function in a Taylor series. In this regard, hyperholomorphic analysis on commutative unitary algebras is a natural extension of complex analysis, despite the fact that in these algebras we have the problem of zero divisors. There are many commutative generalizations of complex numbers, say, hyperbolic numbers, bicomplex algebra etc. [1]. In [2], it is proved that hyperholomorphic functions on bicomplex algebra can be expanded in a Taylor series. In this article, we generalize this result to any finite-dimensional commutative algebra.

*Email: pogor@zu.edu.ua 


\section{Differentiation in finite-dimensional commutative algebra}

Let $\mathbf{A}$ be a finite-dimensional commutative unitary algebra over $K=\mathbb{R}$ (or $\mathbb{C}$ ), a set of vectors $\vec{e}_{0}, \vec{e}_{1}, \ldots, \vec{e}_{n}$ be a basis of $\mathbf{A}$, and $\vec{e}_{0}$ be algebra identity. Consider a function $\vec{f}: \mathbf{A} \rightarrow \mathbf{A}$ of the following form

$$
\vec{f}(\vec{x})=\sum_{k=0}^{n} \vec{e}_{k} u_{k}(\vec{x}),
$$

where $u_{k}(\vec{x})=u_{k}\left(x_{0}, x_{1}, \ldots, x_{n}\right)$ are real (or complex) functions of $n+1$ arguments.

Definition 2.1 $\vec{f}(\vec{x})$ is called A-differentiable at a point $\vec{x}_{0} \in \mathbf{A}$ if there exists the function $\vec{f}^{\prime}: \mathbf{A} \rightarrow \mathbf{A}$ such that for any $\vec{h} \in \mathbf{A}$

$$
\vec{h} \vec{f}^{\prime}\left(\vec{x}_{0}\right)=\lim _{\varepsilon \rightarrow 0} \frac{\vec{f}\left(\vec{x}_{0}+\varepsilon \vec{h}\right)-\vec{f}\left(\vec{x}_{0}\right)}{\varepsilon},
$$

where $\overrightarrow{f^{\prime}}$ doesn't depend on $\vec{h}$.

A function $\vec{f}$ is said to be A-holomorphic if $\vec{f}$ is A-differentiable at every point of $\mathbf{A}$. TheOrem 2.2 A function $\vec{f}(\vec{x})=\sum_{k=0}^{n} \vec{e}_{k} u_{k}(\vec{x})$ A-holomorphic if and only if there exists the function $\vec{f}^{\prime}: \mathbf{A} \rightarrow \mathbf{A}$ such that for all $k=0,1, \ldots, n$, and $\forall \vec{x} \in \mathbf{A}$

$$
\vec{e}_{k} \vec{f}^{\prime}(\vec{x})=\lim _{\varepsilon \rightarrow 0} \frac{\vec{f}\left(\vec{x}+\varepsilon \vec{e}_{k}\right)-\vec{f}(\vec{x})}{\varepsilon},
$$

where $\vec{f}^{\prime}$ does not depend on $\vec{e}_{k}$.

Proof Suppose that (2.2) is fulfilled, then it is easily verified that

$$
\begin{gathered}
\vec{f}^{\prime}=\lim _{\varepsilon \rightarrow 0} \frac{\vec{f}\left(\vec{x}+\varepsilon \vec{e}_{0}\right)-\vec{f}(\vec{x})}{\varepsilon}=\sum_{k=0}^{n} \vec{e}_{k} \frac{\partial u_{k}}{\partial x_{0}}, \\
\vec{e}_{1} \vec{f}^{\prime}=\lim _{\varepsilon \rightarrow 0} \frac{\vec{f}\left(\vec{x}+\varepsilon \vec{e}_{1}\right)-\vec{f}(\vec{x})}{\varepsilon}=\sum_{k=0}^{n} \vec{e}_{k} \frac{\partial u_{k}}{\partial x_{1}}=\vec{e}_{1} \sum_{k=0}^{n} \vec{e}_{k} \frac{\partial u_{k}}{\partial x_{0}}, \\
\vdots \\
\vec{e}_{n} \vec{f}^{\prime}=\lim _{\varepsilon \rightarrow 0} \frac{\vec{f}\left(\vec{x}+\varepsilon \vec{e}_{n}\right)-\vec{f}(\vec{x})}{\varepsilon}=\sum_{k=0}^{n} \vec{e}_{k} \frac{\partial u_{k}}{\partial x_{n}}=\vec{e}_{n} \sum_{k=0}^{n} \vec{e}_{k} \frac{\partial u_{k}}{\partial x_{0}} .
\end{gathered}
$$

Consider $\vec{h}=\sum_{k=0}^{n} h_{k} \vec{e}_{k}$. It follows from equation (2.3) that

$$
\begin{gathered}
h_{0} \vec{f}^{\prime}=h_{0} \sum_{k=0}^{n} \vec{e}_{k} \frac{\partial u_{k}}{\partial x_{0}}, \\
h_{1} \vec{e}_{1} \vec{f}^{\prime}=h_{1} \sum_{k=0}^{n} \vec{e}_{k} \frac{\partial u_{k}}{\partial x_{1}}, \\
\vdots \\
h_{n} \vec{e}_{n} \vec{f}^{\prime}=h_{n} \sum_{k=0}^{n} \vec{e}_{k} \frac{\partial u_{k}}{\partial x_{n}} .
\end{gathered}
$$


This implies that

$$
\begin{aligned}
\vec{h} \vec{f}^{\prime} & =h_{0} \sum_{k=0}^{n} \vec{e}_{k} \frac{\partial u_{k}}{\partial x_{0}}+h_{1} \sum_{k=0}^{n} \vec{e}_{k} \frac{\partial u_{k}}{\partial x_{1}}+\cdots+h_{n} \sum_{k=0}^{n} \vec{e}_{k} \frac{\partial u_{k}}{\partial x_{n}} \\
& =\lim _{\varepsilon \rightarrow 0} \frac{\vec{f}\left(\vec{x}_{0}+\varepsilon \vec{h}\right)-\vec{f}\left(\vec{x}_{0}\right)}{\varepsilon} .
\end{aligned}
$$

Furthermore, it follows from equation (2.3) that

$$
\begin{aligned}
& h_{0} \sum_{k=0}^{n} \vec{e}_{k} \frac{\partial u_{k}}{\partial x_{0}}+h_{1} \sum_{k=0}^{n} \vec{e}_{k} \frac{\partial u_{k}}{\partial x_{1}}+\cdots+h_{n} \sum_{k=0}^{n} \vec{e}_{k} \frac{\partial u_{k}}{\partial x_{n}} \\
& \quad=h_{0} \sum_{k=0}^{n} \vec{e}_{k} \frac{\partial u_{k}}{\partial x_{0}}+h_{1} \vec{e}_{1} \sum_{k=0}^{n} \vec{e}_{k} \frac{\partial u_{k}}{\partial x_{0}}+\cdots+h_{n} \vec{e}_{n} \sum_{k=0}^{n} \vec{e}_{k} \frac{\partial u_{k}}{\partial x_{0}}
\end{aligned}
$$

Therefore, for every $\vec{h} \in A$

$$
\vec{h} \sum_{k=0}^{n} \vec{e}_{k} \frac{\partial u_{k}}{\partial x_{0}}=\lim _{\varepsilon \rightarrow 0} \frac{\vec{f}\left(\vec{x}_{0}+\varepsilon \vec{h}\right)-\vec{f}\left(\vec{x}_{0}\right)}{\varepsilon}
$$

or

$$
\vec{f}^{\prime}=\sum_{k=0}^{n} \vec{e}_{k} \frac{\partial u_{k}}{\partial x_{0}}
$$

By using equation (2.3), we have

$$
\begin{aligned}
\sum_{k=0}^{n} \vec{e}_{k} \frac{\partial u_{k}}{\partial x_{1}}= & \vec{e}_{1} \sum_{k=0}^{n} \vec{e}_{k} \frac{\partial u_{k}}{\partial x_{0}}, \\
\sum_{k=0}^{n} \vec{e}_{k} \frac{\partial u_{k}}{\partial x_{2}}= & \vec{e}_{2} \sum_{k=0}^{n} \vec{e}_{k} \frac{\partial u_{k}}{\partial x_{0}}, \\
& \vdots \\
\sum_{k=0}^{n} \vec{e}_{k} \frac{\partial u_{k}}{\partial x_{n}}= & \vec{e}_{n} \sum_{k=0}^{n} \vec{e}_{k} \frac{\partial u_{k}}{\partial x_{0}} .
\end{aligned}
$$

Equation (2.5) will be called the Cauchy-Riemann type conditions. It follows from Theorem 1.2 that if $\vec{f}(\vec{x})=\sum_{k=0}^{n} \vec{e}_{k} u_{k}(\vec{x})$ satisfies (1.5) then $\vec{f}$ is A-holomorphic.

THEOREM 2.3 If $\vec{f}$ is A-holomorphic and $u_{k} \in C^{\infty}, k=1, \ldots, n$, then for all $l \geq 1$ there exists $\vec{f}^{(l)}$, which is A-holomorphic and $\vec{f}^{(l)}=\sum_{k=0}^{n} \vec{e}_{k}\left(\partial^{l} u_{k} / \partial x_{0}^{l}\right)$.

Proof It is easy to see that functions $u_{k}^{\prime}=\left(\partial u_{k} / \partial \vec{x}_{0}\right), k=1, \ldots, n$, satisfy conditions (2.5) since $u_{k} \in C^{\infty}$. So $\vec{f}^{\prime}$ is A-holomorphic and $\vec{f}^{\prime}=\sum_{k=0}^{n} \vec{e}_{k}\left(\partial^{2} u_{k} / \partial x_{0}^{2}\right)$ (see (2.4)). In complete analogy with this we can show that $\vec{f}^{(l)}$ is A-holomorphic and $\vec{f}^{(l)}=$ $\sum_{k=0}^{n} \vec{e}_{k}\left(\partial^{l} u_{k} / \partial x_{0}^{l}\right)$. 
Theorem 2.4 Suppose $\vec{f}$ is an A-holomorphic function with $u_{k} \in C^{\infty}, k=0,1, \ldots, n$ and for fixed $\vec{x}, \vec{h} \in \mathbf{A}$ there exists $K>0$ such that $\|\vec{f}(l) \vec{h}\| K^{l}$ for $l=1,2, \ldots$ Then $\vec{f}$ can be expanded in a Taylor series

$$
\vec{f}(\vec{x}+\vec{h})=\vec{f}(\vec{x})+\vec{f}^{\prime}(\vec{x}) \vec{h}+\frac{1}{2 !} \vec{f}^{\prime}(\vec{x}) \vec{h}^{2}+\cdots
$$

Proof Consider the function $\vec{F}(t)=\vec{f}(\vec{x}+t \vec{h})$. It is easily verified that $\mathrm{d}^{l} \vec{F}(0) / \mathrm{d} t^{l}=\vec{f}^{l}(\vec{x}) \vec{h}^{l}$. Taking into account that $\left\|\vec{f}^{(l)} \vec{h}\right\| K^{l}$ for $>l=1,2, \ldots$, it follows that the function $\vec{F}(t)$ can be expanded in a Taylor series as follows

$$
\vec{F}(t)=\vec{F}(0)+\sum_{l \geq 1} \frac{1}{l !} \frac{\mathrm{d} l \vec{F}(0)}{\mathrm{d} t^{l}} t^{l} .
$$

Putting $t=1$, we get (2.6).

In the particular case where a bicomplex (or hyperbolic) function is hyperholomorphic and satisfies Theorem 2.4, it can be expanded in a Taylor series $(2.6)[1,2]$.

\section{Zeros of polynomials in commutative algebras}

Since on numerous occasions A-holomorphic function can be approximated by its Taylor polynomial of finite degree, zeros of such functions might be studied if we can calculate zeros of polynomials. Let $p_{m}(w)=a_{m} w^{m}+a_{m-1} w^{m-1}+\cdots+a_{0}$ be a polynomial in the algebra A. Our purpose is to investigate the structure of the set of zeros of the equation

$$
p_{m}(w)=0
$$

Theorem 3.1 If $\mathbf{A}$ has $n+1$ non trivial idempotents $i_{0}, i_{1}, \ldots, i_{n}$ such that $i_{p} i_{r}=0$ for $p \neq r$, and $\sum_{l=0}^{n} i_{l}=1$, then equation 3.1 can be reduced to the system of polynomial equations in the field $K$.

Proof As a preliminary to the proof of the theorem, we shall prove several auxiliary lemmas.

Lemma 3.2 Idempotents $i_{0}, i_{1}, \ldots, i_{n}$ are linearly independent vectors.

Proof Suppose the contrary, then there exist $k_{0}, k_{1}, \ldots, k_{n} \in K$ such that $\sum_{p=0}^{n}\left|k_{p}\right|>0$ and $\sum_{p=0}^{n} k_{p} i_{p}=0$. By using the properties of idempotents, we have $k_{p} i_{p}=0$ for all $p=0,1, \ldots, n$, but this is impossible. Indeed, if $k_{p} i_{p}=0$ for $k_{p} \neq 0$, then $k_{p}^{-1}\left(k_{p} i_{p}\right)=i_{p}=0$.

Denote by $I_{l}=\left\{a i_{l} \mid a \in A\right\}$ the principal ideal generated by $i_{l}, l=0,1, \ldots, n$. It follows from Theorem 2.3 that the algebra $\mathbf{A}$ can be decomposed in the direct sum (the Pierce decomposition): $A=I_{0} \oplus I_{1} \oplus \cdots \oplus I_{n}$.

Lemma 3.3 If $a \in I_{l}$ then there exists $k \in K$ such that $a=k i_{1}$, i.e., the ideal $I_{l}$ can be represented in the following form $I_{l}=\left\{k i_{l} \mid k \in K\right\}$. 
Proof For $a \in I_{l}$ there exists $b \in \mathbf{A}$ such that $a=b i_{l}$. Since $i_{0}, i_{1}, \ldots, i_{n}$ are linearly independent, there exist $k_{0}, k_{1}, \ldots, k_{n} \in K$ such that $b=\sum_{p=0}^{n} k_{p} i_{p}$. Thus, $a=b i_{l}=\left(\sum_{p=0}^{n} k_{p} i_{p}\right) i_{l}=k_{l} i_{l}$.

Let us consider decompositions

$$
\begin{aligned}
a_{r} & =a_{r}^{(0)}+\cdots+a_{r}^{(n)}, \quad r=0,1, \ldots m, \\
w & =w_{0}+\cdots+w_{n},
\end{aligned}
$$

where $a_{r}^{(p)}, w_{p} \in I_{p}$. Plugging (3.2) into (3.1), we obtain the following system of polynomial equations

$$
\begin{gathered}
a_{m}^{(0)} w_{0}^{m}+a_{m-1}^{(0)} w_{0}^{m-1}+\cdots+a_{0}^{(0)}=0, \\
a_{m}^{(1)} w_{1}^{m}+a_{m-1}^{(1)} w_{1}^{m-1}+\cdots+a_{0}^{(1)}=0, \\
\vdots \\
a_{m}^{(n)} w_{n}^{m}+a_{m-1}^{(n)} w_{n}^{m-1}+\cdots+a_{0}^{(n)}=0 .
\end{gathered}
$$

It follows from Lemma 1.3 that $a_{r}^{(s)}=k_{r}^{(s)} i_{s}, w_{s}^{r}=x i_{s}$, where $k_{r}^{(s)}, x \in K$.

Therefore, taking $i_{s}$ out of the expression $a_{m}^{(s)} w_{s}^{m}+a_{m-1}^{(s)} w_{s}^{m-1}+\cdots+a_{0}^{(s)}=$ $0, s=0, \ldots, n$, the system (3.3) can be reduced to the system of $n+1$ polynomial equations in $K$ with coefficients $k_{r}^{(s)}$.

Example 3.4 Let $\mathbf{A}$ be the bicomplex algebra, i.e., $\mathbf{A}=\left\{c_{0}+e c_{1} \mid c_{0}, c_{1} \in \mathbb{C}\right\}$, where $e^{2}=1$ and $\mathbf{A}$ is commutative. The bicomplex algebra has two idempotents $i_{0}=(1+e) / 2$ and $i_{1}=(1-e) / 2$. It is easy to see that $i_{0} i_{1}=0$ and $i_{0}+i_{1}=1$. Thus, in this case polynomial equation (3.1) can be reduced to the system of two polynomial equations in $\mathbb{C}$ [3]. Many properties of quarternionic polynomials have been presented in [4].

Example 3.5 Suppose $\mathbf{A}$ is the commutative algebra of the following form $A=\left\{a_{0}+e a_{1}+f a_{2}+g a_{3} \mid a_{k} \in \mathbb{R}\right\}$, where $e^{2}=f^{2}=g^{2}=1$ and $e f g=1$. This algebra has four idempotents: $i_{0}=(1+e+f+g) / 4, i_{1}=(1-e-f+g) / 4, i_{2}=(1+e-f-g) / 4$, $i_{3}=(1-e+f-g) / 4$. It is easy to see that $i_{k} i_{l}=0$ for $k \neq l$, and $i_{0}+i_{1}+i_{2}+i_{3}=1$. Therefore, in this case polynomial equation (3.1) can be reduced to the system of four polynomial equations in $\mathbb{R}$.

\section{References}

[1] Olariu, S., 2002, Complex Numbers in n Dimensions. North-Holland Mathematics Studies, Vol. 190 (Amsterdam-Boston: Elsevier).

[2] Rönn, S., 2001, Bicomplex algebra and function theory. arXiv:math.CV/0101200v1, 1, 1-71.

[3] Pogorui, A.A. and Rodriguez-Dagnino, R.M., 2006, On the set of zeros of bicomplex polynomials. Complex Variables and Elliptic Equations, 51(7), 725-730.

[4] Pogorui, A.A. and Shapiro, M., 2004, On the structure of the set of the zeros of quaternionic polynomials. Complex Variables, Theory and Application, 49(6), 379-389. 
Copyright of Complex Variables \& Elliptic Equations is the property of Taylor \& Francis Ltd and its content may not be copied or emailed to multiple sites or posted to a listserv without the copyright holder's express written permission. However, users may print, download, or email articles for individual use. 